\title{
Understanding the Use of Electronic Means to Seek Personal Health Information Among Adults in the United States
}

\author{
Noara Alhusseini ${ }^{1}$, Jim E. Banta ${ }^{2}$, Jisoo $\mathrm{Oh}^{2}$, Susanne Montgomery ${ }^{2}$ \\ 1. Public Health, Alfaisal University, Riyadh, SAU 2. Public Health, Loma Linda University, Loma Linda, USA
}

Corresponding author: Noara Alhusseini, alhusseini.n@gmail.com

\begin{abstract}
In this study, we explored who is most likely to use electronic means to seek health information and why; our research was guided by the Health Belief Model (HBM). We used the National Cancer Institute's Health Information Trends Survey (HINTS) dataset for 2017 and $2018(\mathrm{n}=6,697)$. We found that $67.5 \%$ of US adults used electronic means to seek health information and that females (52.4\%), non-Hispanic whites (63.8\%), those with at least some college education (76.5\%), and those with a household income of at least \$50,000 per year (58.3\%) were most likely to do so. Respondents reporting depression were $42 \%$ more likely to use electronic means to seek health information, suggesting that stigma about mental health may direct people with depression to seek online information to avoid face-to-face communication. Using a tablet to track progress on a health-related goal [odds ratio $(\mathrm{OR})=2.38, \mathrm{p}<0.0001$ ], and using a tablet to make a decision about treating an illness $(\mathrm{OR}=6.00, \mathrm{p}<0.0001)$ were highly associated with seeking electronic health information. As the internet remains largely unregulated, this suggests that health systems link their patients to trustworthy resources for preventive and treatment-related information, since many already engage in internet-guided health information-seeking.
\end{abstract}

Categories: Medical Education, Public Health, Healthcare Technology

Keywords: health information, internet, electronics, health belief model, depression, hints

\section{Introduction}

The internet is comprised of interconnected, global networks that facilitate communication and access to a variety of information [1]. This is achieved with a broad range of channels including websites, blogs, and social media networks that provide user-generated content [2]. Over three billion adults use the internet worldwide for communication and $90 \%$ of them use it to access information [3,4]. Information-seeking refers to the purposive search for information to satisfy a goal [5]. Seeking online health information is the third most common online activity among the US population [4] with $74 \%$ of adults using the web and $80 \%$ of them searching for health-related information [6], mostly about a specific disease (66\%) or treatment plan (56\%) [4].

Review began 09/28/2020 Review ended 10/26/2020 Published 10/27/2020

\section{(๑) Copyright 2020}

Alhusseini et al. This is an open access article distributed under the terms of the Creative Commons Attribution License CC-BY 4.0., which permits unrestricted use, distribution, and reproduction in any medium, provided the original author and source are credited.
The internet is considered a rich source of information and has a large online medical resource, containing over 100,000 health-related websites. It contains formal and informal sources allowing for health information dissemination in a fast, easy, and accessible way [5]. With the development of electronic health platforms, a plethora of new opportunities for individuals to communicate, access, and exchange healthrelated information have emerged. Many electronic sources such as mobile devices, computers, tablets, smartphones, and electronic book devices enable users to connect to the internet to seek health information [7].

More recently, the internet has become a vital source for individuals who seek health-related information [8]. There are many factors that encourage individuals to seek health-related information. The increased availability of online medical information, its cost-effectiveness, and the ability to tailor the information based on users' needs highly motivate internet users to use the web to seek health-related information. In addition, individuals with an increased desire to take more responsibility for their health are attracted to the internet because it allows finding instant information that may guide their behaviors and lifestyles. People who use the internet to look up information are called internet surfers. They consider the internet a useful instrument to increase health awareness by finding support from healthcare providers and other users who share medical experiences.

\section{Seeking health information}

To better understand individuals' behaviors when seeking online health information, researchers have examined patients' behaviors. For example, Caiata-Zufferey et al. (2010) confirmed that patients often use the internet before and after a medical encounter. Before a consultation, patients use online sources to assess their needs, choose the right healthcare provider, and prepare for the consultation. After a consultation, they go online to understand their diagnosis, treatment plans, and look for alternative medical 
options. Seeking online health information can also be driven by patients' dissatisfaction with the consultation or a desire to avoid bothering healthcare providers [9].

For many, the internet is considered a useful tool to research information because it has many resources that match the needs of diverse consumers. Internet users seek online support for medical-related information. People use the web to search for certain medical conditions to increase knowledge, find emotional support, and exchange experiences. Users are highly motivated because they can limit their search and find answers rapidly to health-related queries and concerns. It also allows access and contributions from all users equally, regardless of background, race, or age. Every user gets a chance to search freely and express his/her opinion without discrimination [2]. Therefore, online consumers consider the internet a popular source of health information. The vastness of health information on the internet attracts consumers to use it, especially in the setting of a global push for consumer-focused healthcare [10].

There are also a number of pitfalls to seeking health information on the internet. With few checks and balances and a general lack of universal quality requirements for publishing health-related content, the internet can provide unreliable, inconsistent, and at times misleading medical information $[2,10,11]$. The volume of available health information on the internet can result in information overload. In addition, the excessive volume of irrelevant information obtained from complex search engines and the technical language involved often cause confusion in lay consumers [10].

\section{Utilization of theory in the context of health information-seeking on the internet}

It is well established that interventions guided by theories are more effective in promoting change. Since many consumers already use the internet as part of personally guided efforts to consider behavior changerelated decision-making, it would suggest that taking a theoretical lens to the issue may be of benefit. The Health Belief Model (HBM) has been noted to be useful in advancing the efficacy of intervention both online and offline [12]. Using a theoretical lens like that of the HBM may assist in this regard as the HBM could aid in advancing the efficacy of online and offline health promotion intervention. According to Glanz et al. (2015), individuals are likely to engage in a health-related behavior if they think that they are susceptible to a condition, see the condition as serious, believe that there is benefit in that behavior (such as informationseeking via the internet), that the perceived costs or barriers are outweighed by the benefits, and believe that they can do this efficaciously [13].

\section{Purpose of the study}

Seeking health information on the internet and social media has become common among adults in the United States. Researchers are still uncertain about using online health interventions and changes in health behaviors. In addition, there is limited research exploring the use of electronic methods to seek health information from a theoretical framework perspective to understand healthy behaviors to manage chronic diseases. There is a clear gap in the literature on a national level to understand and explore the phenomenon of using the internet and social media to seek health information.

Since there is a lack of research data about using electronic means to look for health information, we used variables aligned with conceptual components of the HBM to explore using electronic means to seek health information including perceived susceptibility, perceived severity, perceived benefits, and perceived selfefficacy.

\section{Research question}

Our study focused on addressing the following question: among adults in the United States, do the components of the HBM as measured by perceived susceptibility, severity, benefits, and self-efficacy predict using electronic devices to seek health information within the past 12 months?

Sub-Questions

This will be accomplished by answering the following sub-questions: does perceived susceptibility predict using electronic devices to seek health information within the past 12 months among adults in the United States? Does perceived severity predict using electronic devices to seek health information within the past 12 months among adults in the United States? Do perceived benefits predict using electronic devices to seek health information within the past 12 months among adults in the United States? Does perceived selfefficacy to take care of one's health predict using electronic devices to seek health information within the past 12 months among adults in the United States? What is the relative contribution of each conceptual element of the HBM in explaining using electronic means to look for health information?

\section{Materials And Methods}

The Health Information Trends Survey (HINTS) dataset was used in this study. A secondary analysis was conducted using HINTS 5, cycle 1 of 2017, and HINTS 5, cycle 2 of 2018. The HINTS is a nationally 
representative survey that has been administered by the National Cancer Institute (NCI) since 2003. It is freely available for public use. The Institutional Review Board at Loma Linda University approved this study. The target population consisted of civilian non-institutionalized adults aged 18 or older in the United States. The HINTS program collects data on the needs for, access to, and use of health-related information and health-related behaviors and knowledge among Americans. The data were collected using a selfadministered mail questionnaire. The dataset 5 cycle 1 of 2017 consisted of 3,285 respondents and the dataset of 2018 consisted of 3,504 respondents. The two datasets were merged to obtain a total of 6,697 respondents. Ninety-two surveys were dropped due to missing data for the outcome measure.

HINTS exclusion criteria included adolescents, children, homeless people, and institutionalized populations. Every sampled participant who completed the survey received a full sample weight, which was used to calculate population and subpopulation estimates. Computation of the full-sample weights comprised the following phases: calculating household level base weights, adjusting for household nonresponse, calculating person-level initial weights, and calibrating the person-level weights to population counts. The replicate weights were calculated using the jackknife replication method [14].

Our outcome measure was "using electronic means to seek health information" measured by addressing the following question: "In the past 12 months, have you used a computer, smartphone, or other electronic means to do the following: look for health or medical information for yourself? (Yes/no)." We then reviewed the existing standardized HINTS survey to identify variables we could align with components of the HBM. For perceived susceptibility, we used the following question: “In general, would you say your health is?” The answer choices included excellent, very good, good, fair, and poor. Six variables were assigned to perceived severity. The survey questions were: "Has your doctor or other health professional ever told you that you had any of the following medical conditions: diabetes or high blood sugar; high blood pressure or hypertension; a heart condition such as heart attack, angina, or congestive heart failure; chronic lung disease, asthma, emphysema, or chronic bronchitis; arthritis or rheumatism; and depression or anxiety disorder." The answer choices for the questions were (yes/no). For perceived benefits, two variables were assigned. The first question was as follows: "Has your tablet or smartphone helped you track progress on a health-related goal such as quitting smoking, losing weight, or increasing physical activity?" and the second question was, "Has your tablet or smartphone helped you make a decision about how to treat an illness or condition?" The answer choices were (yes/no).

For self-efficacy, one variable was identified: “Overall, how confident are you about your ability to take good care of your health?” The answer choices included: completely confident, very confident, somewhat confident, a little confident, and not confident at all. Demographic variables included age with five categories: gender (male, female), race/ethnicity (non-Hispanic white, non-Hispanic black, Hispanic, nonHispanic other); education with five categories: less than high school, high school graduate, some college, bachelor's degree, and post-baccalaureate degree; household income with four categories: \$0-19,999, $\$ 20,000-49,999, \$ 50,000-99,999$, and $\$ 100,000$ or more; and employment with three categories: employed, retired, and other.

We conducted a series of chi-squared tests to study the relationship between electronic means to seek health information and demographics (Table 1). We then created five models for logistic regression analysis to determine the model fit. Model 1 explored the effect of demographics on the outcome variable, using electronic means to seek health information. Model 2 included the outcome and the first conceptual component of the HBM, perceived susceptibility. Model 3 included the outcome and the second conceptual component, perceived severity. Model 4 included the outcome and the third conceptual component, perceived benefits. Model 5 included the outcome and the fourth conceptual component, perceived selfefficacy. Then, a final model was created with only those variables that were found statistically significant in the previous five models.

\section{Results}

As shown in Table 1, 6,697 respondents completed the survey, representing an estimated annual 245.8 million adults in the United States. More than two-thirds of the respondents (67.5\%) had used electronic means to seek health information for themselves. Among those who did, the majority $(58.8 \%)$ were between 35 and 64 years of age; $52.4 \%$ were female, most were non-Hispanic whites (63.8\%), and most had at least some college education (76.5\%). Household income for the majority (58.3\%) of personal health information seekers was at least $\$ 50,000$ per year and the majority (63\%) of them were employed. Those who did not use electronic means to seek personal health information were older individuals: $64.2 \%$ were 50 years of age or older; $51 \%$ were high school graduates or less, and $52.6 \%$ had a household income of less than $\$ 50,000$ per year. All comparisons were statistically significant ( $p<0.0001)$.

\section{Using electronic means to seek health information} Total

Age (years)
Yes, $\mathbf{n}(\%)$

4,523
No, $n(\%)$

2,174 


\section{Cureus}

\begin{tabular}{|c|c|c|c|}
\hline $18-34$ & $669(26.3)$ & $97(11.9)$ & \\
\hline $35-49$ & $1,082(30.5)$ & $222(18.7)$ & \\
\hline $50-64$ & $1,522(28.3)$ & $635(32.5)$ & \\
\hline $65-74$ & $836(9.3)$ & $553(14.5)$ & \\
\hline $75+$ & $320(3.9)$ & $545(17.2)$ & \\
\hline Missing & $93(1.7)$ & $122(5.2)$ & \\
\hline Gender & & & $<0.0001$ \\
\hline Male & $1,741(46.7)$ & $923(51.8)$ & \\
\hline Female & $2,727(52.4)$ & $1,189(45.5)$ & \\
\hline Missing & $55(0.9)$ & $62(2.7)$ & \\
\hline Race/ethnicity & & & $<0.0001$ \\
\hline Non-Hispanic white & $2,788(63.8)$ & $1,035(52.0)$ & \\
\hline Non-Hispanic black & $513(9.1)$ & $325(11.1)$ & \\
\hline Hispanic & $551(13.8)$ & $323(16.5)$ & \\
\hline Non-Hispanic other & $385(8.4)$ & $121(5.9)$ & \\
\hline Missing & $286(4.8)$ & $370(14.5)$ & \\
\hline Education & & & $<0.0001$ \\
\hline Less than high school & $161(5.3)$ & $315(16.7)$ & \\
\hline High school graduate & $583(17.0)$ & $644(34.3)$ & \\
\hline Some college & $1,318(37.5)$ & $639(31.9)$ & \\
\hline Bachelor's degree & $1,407(24.0)$ & $318(8.8)$ & \\
\hline Post-baccalaureate & $988(15.0)$ & $178(5.6)$ & \\
\hline Missing & $66(1.2)$ & $80(2.7)$ & \\
\hline Household income & & & $<0.0001$ \\
\hline$\$ 0-19,999$ & $517(11.7)$ & $599(25.7)$ & \\
\hline$\$ 20,000-49,999$ & $1,018(22.4)$ & $604(26.9)$ & \\
\hline$\$ 50,000-99,999$ & $1,379(30.5)$ & $431(21.6)$ & \\
\hline$\$ 100,000$ or more & $1,240(27.8)$ & $194(11.9)$ & \\
\hline Missing & $369(7.5)$ & $346(14.0)$ & \\
\hline Employment & & & $<0.0001$ \\
\hline Employed & $2,597(63.0)$ & $658(39.1)$ & \\
\hline Retired & $1,109(13.5)$ & $937(28.5)$ & \\
\hline Other & $696(21.1)$ & $436(25.5)$ & \\
\hline Missing & $121(2.4)$ & $143(6.9)$ & \\
\hline
\end{tabular}

TABLE 1: Survey-weighted sociodemographic characteristics of HINTS respondents, 2017-2018 $(n=6,697)$

HINTS: Health Information Trends Survey 


\section{Cureus}

the conceptually aligned HBM variables. Most respondents reported at least very good health (51.1\%) with no chronic conditions. Using electronic means to make a decision about treating an illness $(42.1 \%)$ or using electronic means to track progress on a health-related goal (46.8\%) was significantly related to seeking electronic health information $(\mathrm{p}<0.0001)$. While most reported being at least somewhat confident in taking care of themselves (94.9\%), this was not significantly related to seeking electronic health information. For perceived severity, i.e. having a serious health diagnosis and its relationship to seeking health information, all chronic diseases except lung disease were significantly related to seeking electronic health information. However, those with hypertension or a mental health diagnosis were most likely to use the internet to seek health information: $32.5 \%$ and $24.7 \%$ respectively.

\begin{tabular}{|c|c|c|c|}
\hline Using electronic means to seek health information & Yes, $\mathbf{n}(\%)$ & No, $n(\%)$ & P-value \\
\hline Total & 4,523 & 2,174 & \\
\hline Perceived susceptibility & & & 0.0003 \\
\hline \multicolumn{4}{|l|}{ General health } \\
\hline Excellent & $562(13.1)$ & $208(13.1)$ & \\
\hline Very good & $1,734(38.0)$ & 693 (31.6) & \\
\hline Good & $1,539(34.7)$ & 774 (33.3) & \\
\hline Fair & $545(11.6)$ & $392(17.0)$ & \\
\hline Poor & $98(2.0)$ & $82(4.1)$ & \\
\hline Missing & $37(0.6)$ & $24(0.8)$ & \\
\hline Perceived severity & & & $<0.0001$ \\
\hline \multicolumn{4}{|l|}{ Diabetes/high blood sugar } \\
\hline Yes & 839 (14.5) & $530(21.8)$ & \\
\hline No & $3,610(84.1)$ & $1,571(75.0)$ & \\
\hline Missing & $74(1.4)$ & $73(3.2)$ & \\
\hline Hypertension/high blood pressure & & & $<0.0001$ \\
\hline Yes & $1,840(32.5)$ & $1,158(44.7)$ & \\
\hline No & $2,614(66.2)$ & 945 (52.4) & \\
\hline Missing & $69(1.3)$ & $71(2.9)$ & \\
\hline Heart condition & & & 0.0003 \\
\hline Yes & $364(6.1)$ & 295 (10.2) & \\
\hline No & $4,100(92.8)$ & $1,824(87.8)$ & \\
\hline Missing & $59(1.1)$ & $55(2.1)$ & \\
\hline Chronic lung disease & & & 0.1 \\
\hline Yes & $572(10.7)$ & $321(13.1)$ & \\
\hline No & $3,889(87.9)$ & $1,801(84.8)$ & \\
\hline Missing & $62(1.3)$ & $52(2.1)$ & \\
\hline Arthritis & & & $<0.0001$ \\
\hline Yes & $1,222(19.0)$ & $812(29.0)$ & \\
\hline No & $3,233(79.5)$ & $1,307(68.7)$ & \\
\hline Missing & $68(1.5)$ & $55(2.3)$ & \\
\hline Depression or anxiety disorder & & & 0.01 \\
\hline Yes & $1,108(24.7)$ & 407 (19.) & \\
\hline
\end{tabular}




\section{Cureus}

No

Missing

Perceived benefits

Track progress

Yes

No

Missing

Not applicable

Decision about treating illness

Yes

No

Missing

Not applicable

Self-efficacy

Confidence in self-care

Completely confident

Very confident

Somewhat confident

A little confident

Not confident at all

Missing
3,336 (73.6)
1,705 (78.3)
79 (1.7)
62 (2.4)

$<0.0001$

$\begin{array}{ll}1,956(46.8) & 223(12.8) \\ 2,182(46.9) & 1,061(50.9) \\ 62(1.0) & 84(3.1) \\ 261(4.4) & 656(27.6)\end{array}$

$<0.0001$

1,851 (42.1)

142 (7.7)

2,277 (51.5)

1,131 (55.9)

72 (1.1)

96 (3.2)

262 (4.4)

$665(28.0)$

TABLE 2: Survey-weighed descriptive measures of the Health Belief Model conceptual framework, stratified by using electronic means to seek health information

Logistic regression results are shown in Table 3, Table 4. Due to the list-wise deletion of observations with missing data, the number of observations used in the regression models was $n=4,930$. As shown in model 1 (demographics only) of Table 3, the 18-34 age group was 2.2 times more likely to use electronic means to seek health information compared to the reference age group category (50-64 years; $\mathrm{p}=0.0007$ ). Males were $27 \%$ less likely to use electronics to seek health information than females $(p=0.02)$. Post-baccalaureate degree holders were three times more likely to use electronic means to seek health information compared to high school graduates ( $<<0.0001$ ), and those earning $\$ 100,000$ or more per year were 2.5 times more likely to use electronic means than the reference category $(\$ 0-19,999$ per year; $p=0.0003)$.

\begin{tabular}{|c|c|c|}
\hline Models & Odds ratio & P-value \\
\hline \multicolumn{3}{|l|}{ Model 1} \\
\hline \multicolumn{3}{|c|}{ Age (years) } \\
\hline 18-34 & 0.2 & 0.0007 \\
\hline $35-49$ & 1.6 & 0.004 \\
\hline 50-64 & Reference & \\
\hline 65-74 & 0.71 & 0.04 \\
\hline $75+$ & 0.26 & $<0.0001$ \\
\hline
\end{tabular}




\section{Cureus}

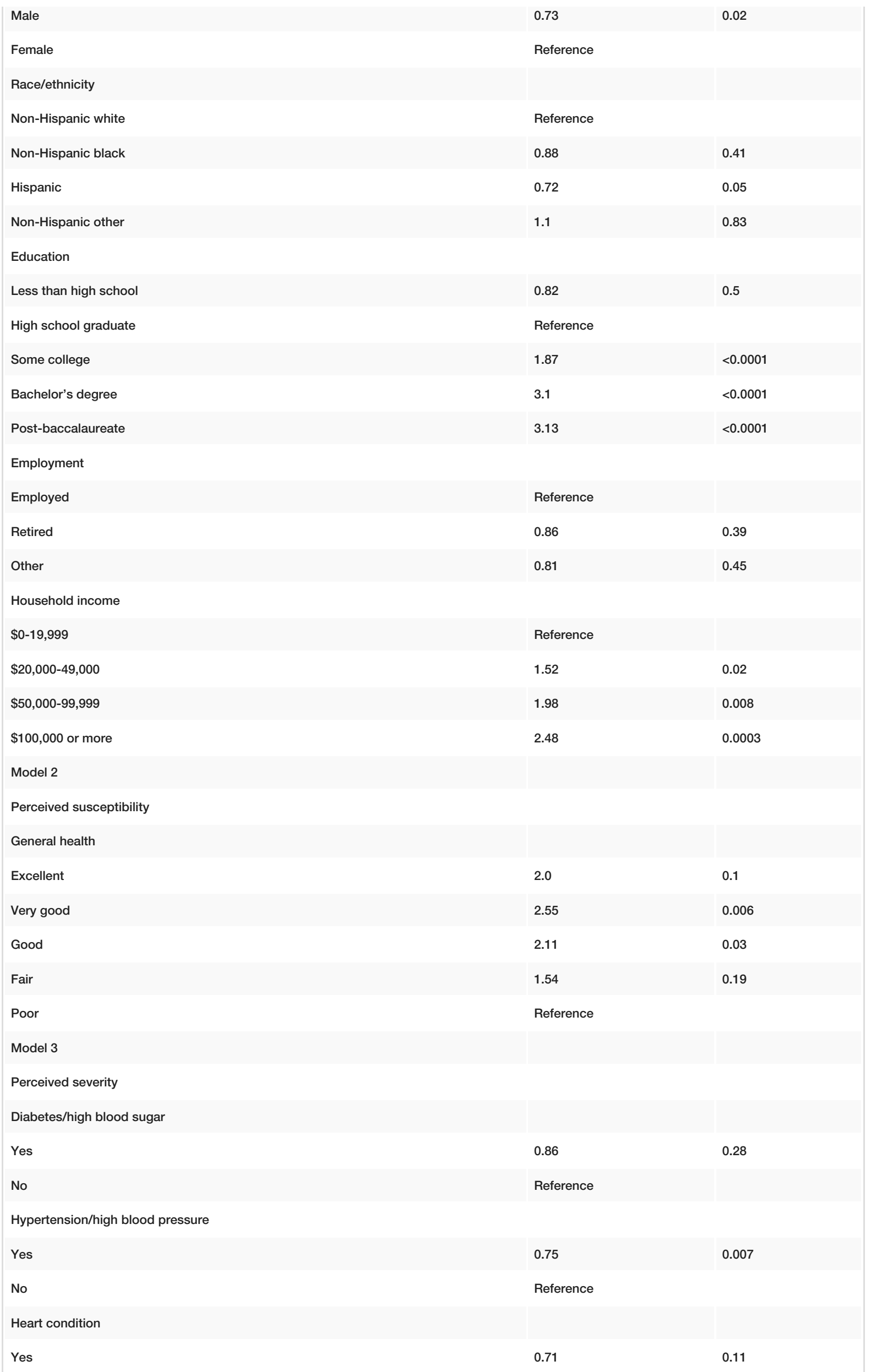




\section{Cureus}

Chronic lung disease

Depression or anxiety disorder

\section{Model 4}

Perceived benefits

Track progress

\section{Model 5}

Self-efficacy

Confidence in self-care

In terms of conceptual variables of the HBM, model 2, exploring perceived susceptibility (perceived health), showed that those who reported having very good health were 2.6 times more likely to use electronic means to seek health information ( $\mathrm{p}=0.006$ ). Model 3 focused on perceived severity and showed that those diagnosed with hypertension/high blood pressure were $25 \%$ less likely and those with arthritis were $30 \%$ less likely to seek health information than those who did not have these diagnoses; however, those diagnosed with depression or anxiety disorder were $65 \%$ more likely to use electronic means to seek health information compared to those who did not have the condition ( $\mathrm{p}=0.0003$ ). Model 4 focused on perceived benefits and concluded that respondents who felt that it was beneficial to use tablets to track progress on a healthrelated goal were 3.5 times more likely to use electronics to seek health information and those who intended to use it to make a decision about treating an illness were 6.3 times more likely to use electronic means to seek health information $(\mathrm{p}<0.0001)$. Model 5 showed that self-efficacy did not significantly predict the use of electronics to seek health information.

The receiver operator curve (ROC) was used to determine the true positive rate (sensitivity) and the false positive rate (specificity) of the HBM components in Table 3. C-statistics for perceived susceptibility (model 


\section{Cureus}

2) was 0.54 , perceived severity (model 3 ) was 0.59 , and perceived self-efficacy (model 5) was 0.5 , which indicates that these models had no or weak predictive value for using electronic means to seek health information. However, the C-statistics value for perceived benefits (model 4) was 0.74, which indicates a good predictive model for using electronic means to seek health information.

Table 4 presents the full regression model, which showed that those who are 75 years of age or above are $63 \%$ less likely to use electronic means to seek health information compared to those who are 50-64 years of age. Those who had at least a bachelor's degree were at least 3.4 times more likely to use electronic means to seek health information compared to high school graduates. Those with an income of $\$ 100,000$ or more per year were 2.8 times more likely to use electronic means to seek health information $(\mathrm{p}<.0001)$. For the conceptual component of perceived severity, those who have depression were $42 \%$ more likely to use electronic means to seek health information, indicating that perceived severity predicted the use of electronics to seek health information at a higher rate. Those with higher perceived benefits because of their intent to track their health-related goals were 2.4 times more likely to use electronics to seek health information ( $\mathrm{p}<0.0001)$, and those needing to make a decision about treating an illness were six times more likely to use electronic means to seek health information.

\begin{tabular}{|c|c|c|c|}
\hline Variables & Odds ratio & $95 \% \mathrm{Cl}$ & P-value \\
\hline \multicolumn{4}{|l|}{ Age (years) } \\
\hline $18-34$ & 1.74 & $(1.06-2.85)$ & 0.03 \\
\hline $35-49$ & 1.33 & (0.93-1.92) & 0.12 \\
\hline $50-64$ & \multicolumn{3}{|l|}{ Reference } \\
\hline $65-74$ & 0.88 & (0.64-1.22) & 0.44 \\
\hline $75+$ & 0.37 & $(0.27-0.51)$ & \\
\hline \multicolumn{4}{|l|}{ Gender } \\
\hline Male & 0.93 & $(0.7-1.23)$ & 0.62 \\
\hline Female & Reference & & \\
\hline \multicolumn{4}{|l|}{ Education } \\
\hline Less than high school & 0.82 & $(0.45-1.5)$ & 0.52 \\
\hline High school graduate & \multicolumn{3}{|l|}{ Reference } \\
\hline Some college & 1.9 & $(1.45-2.47)$ & \\
\hline Bachelor's degree & 3.39 & $(2.37-4.84)$ & \\
\hline Post-baccalaureate & 3.24 & $(2.24-4.69)$ & \\
\hline \multicolumn{4}{|l|}{ Household income } \\
\hline$\$ 0-19,999$ & Reference & & \\
\hline$\$ 20,000-49,999$ & 1.63 & $(1.17-2.29)$ & 0.004 \\
\hline$\$ 50,000-99,999$ & 2.21 & $(1.47-3.33)$ & 0.0001 \\
\hline$\$ 100,000$ or more & 2.82 & $(1.69-4.70)$ & \\
\hline \multicolumn{4}{|l|}{ Perceived susceptibility } \\
\hline \multicolumn{4}{|l|}{ General health } \\
\hline Excellent & 0.92 & $(0.31-2.68)$ & 0.88 \\
\hline Very good & 1.67 & $(0.65-4.28)$ & 0.28 \\
\hline Good & 1.98 & $(0.78-5.07)$ & 0.15 \\
\hline Fair & 2 & $(0.81-4.95)$ & 0.14 \\
\hline Poor & Reference & & \\
\hline Perceived se & & & \\
\hline
\end{tabular}




\section{Cureus}

\begin{tabular}{|c|c|c|c|}
\hline \multicolumn{4}{|c|}{ High blood pressure } \\
\hline Yes & 1.04 & $(0.81-1.34)$ & 0.77 \\
\hline No & Reference & & \\
\hline \multicolumn{4}{|c|}{ Arthritis } \\
\hline Yes & 1.05 & $(0.79-1.38)$ & 0.75 \\
\hline No & Reference & & \\
\hline \multicolumn{4}{|c|}{ Depression or anxiety } \\
\hline Yes & 1.42 & $(1.02-1.97)$ & 0.04 \\
\hline No & Reference & & \\
\hline \multicolumn{4}{|c|}{ Perceived benefits } \\
\hline \multicolumn{4}{|c|}{ Track progress } \\
\hline Yes & 2.38 & (1.68-3.39) & \\
\hline No & Reference & & \\
\hline \multicolumn{4}{|c|}{ Decision about treating illness } \\
\hline Yes & 6 & $(4.11-8.73)$ & \\
\hline No & Reference & & \\
\hline
\end{tabular}

TABLE 4: Regression model for using electronic means to seek health information, selecting only significant findings from Table $3(n=4,930)$

\section{Discussion}

Compared to previously published studies, HINTS respondents reported slightly lower electronic use specifically for health information (67.5\% vs. 80\%) [15], likely because the questions were framed specifically to using the internet to seek personal health information and not more generally, such as searching for self and others. Not surprisingly, non-Hispanic whites, younger respondents, those with higher income and education, and females were more likely to seek personal electronic health information. Similar to our findings, Robertson-Lang et al. (2011) have suggested that individuals with high education, high income, and those under the age of 65 are more likely to search for online health information, indicating the existence of a digital divide [15]. The digital divide separates people who have access to and use the internet from those who do not and parallels health disparity patterns in many other forms [16]. Renahy et al. (2010) indicated that online health information mostly benefits privileged individuals in terms of socioeconomic status, general health, and healthcare access. Inequalities in internet access create obstacles to taking full advantage of using the internet to seek health information. Individuals with low socioeconomic status, a minority race, older age, poor health, and those living in isolated geographical regions are less likely to have internet access [16].

With little additional descriptive information about electronic health information-seeking available, it was of interest that the most significant predictors were perceived severity and perceived benefits. However, not every serious diagnosis predicts more online use. Those who have depression were $42 \%$ more likely to use electronic means to seek health information, but those diagnosed with heart disease and arthritis were actually less likely to seek electronic health information. We also found that even controlling for demographics, individuals who use their tablets or smartphones to track progress on a health-related goal were 2.4 times more likely to use electronic means to seek health information. Moreover, users who find their tablets or smartphones helpful in making a decision about treating a health condition were six times more likely to use electronic means to seek health information. Using a tablet or phone suggest increased levels of comfort with electronic means, which likely affected respondents' willingness to then also seek personal preventive and health information guidance. This aligns with other studies that found that one's belief in the effectiveness of a proposed behavior predicts the likelihood of engaging in that behavior $[17,18]$.

One of our most important findings is that those diagnosed with a mental health condition were $42 \%$ more likely to seek health information. This is likely due to the prevailing societal stigma about mental health, making the internet an attractive and seemingly safe way to seek information and guidance about their condition, which supports the finding [19] that people with depression or anxiety disorders prefer seeking online information in order to avoid talking about their problems or facing health providers and the 
finding [20] that people who are prone to anxiety may be more inclined to seek online health information. It is interesting, however, that individuals diagnosed with hypertension and arthritis were significantly less likely to seek health-related internet information, potentially a sign of their comfort in the treatment recommendations of their physicians, given the normalcy of these commonly diagnosed conditions and the age factor.

Ahadzadeh et al. (2015) collected data from educated, married women living in the state of Selangor, an urbanized state in Malaysia, who had internet access $(\mathrm{n}=293)$. The results showed that perceived health risk and health consciousness had a positive influence on health-related internet use. The perceived usefulness of online sources and attitudes toward internet use for health purposes partially facilitated the influence of health consciousness that could be gained from health-related internet use. The effect of perceived health risk on health-related internet use was fully mediated by the perceived usefulness of the internet and attitude towards its use. In the case of mental health diagnosis, our respondents may have perceived the internet to be more useful and necessary (given stigma and access challenges) than in the case of more mainstream diseases. Thus, engaging in health-related internet use is considered a proactive behavior rather than a reactive one [5]. Our findings mirrored this study as individuals who showed the perceived health risk of depression/anxiety and who found benefits in using electronic means to seek health information by tracking progress on a health-related goal or making a decision in treating illness were more likely to search online for health information.

Kim and Park (2012) conducted a study to develop a model to describe the behavioral intention and the health behavior of health-related information technology consumers. They developed a cross-sectional descriptive correlational design, using the Technology Acceptance Model (TAM) with additional mediating variables from the HBM and the Theory of Planned Behavior. The results showed that perceived threat, perceived usefulness, and perceived ease of use significantly affected users' health-related attitude and behavioral intention. An Individual's health condition, health belief, and health information technology self-efficacy had a significant impact on attitude and behavioral intention through the mediators of perceived threat, usefulness, and ease of use [18]. Their study confirms our findings as respondents who found perceived benefits of using electronics were significantly more likely to use electronic means to seek health information for a disease that may have represented a more personal threat so that they sought treatment options or that they were reluctant to share given stigma.

The level of understanding of patient health information may be a predictor of health behavior and the use of health services. Individuals who frequently seek health information tend to participate in their care management. Informed patients use the internet as a useful informational tool to gain an understanding of symptoms and medical conditions or read online health interventions [21]. For instance, Tate et al. (2015) encourage using online sources to participate in physical activity interventions that aim at reducing body weight by increasing motivation levels [22]. Internet-based exercise interventions that provide exercise coaching and goal-setting can result in increased physical activity engagement [23]. Our findings provide a better understanding of people's behavior, which can help in improving online health interventions.

Due to the cross-sectional nature of the study, it was not possible to infer causal relationships between variables in the study. The response rate for both datasets was $32 \%$, which is considered relatively low and may have created a non-response bias. In addition, using existing datasets and merging two datasets limited the choices related to variables. Since HINTS was not optimized for HBM research, we could only do our best to align available variables to the model. This may be the reason that, contrary to the model prediction, we found only the perceived severity and perceived benefits components of the HBM to be significantly related to our outcome. However, given the increasing importance of internet-based health informationseeking, more research exploring theory-guided profiles of those availing themselves of this approach is needed.

\section{Conclusions}

Our findings suggest targeted framing of online health information, as those with plans for preventive behavior, those facing a serious illness, and those with mental health needs are more likely to use the internet for health-specific information. As the internet remains largely unregulated, with few quality controls, it is critical that health systems link their patients to trustworthy resources for preventive information as well as treatment-related information, since many already engage in internet-guided health information-seeking.

Seeking health information online is a relatively new approach for gaining health knowledge, changing health behaviors, and improving health outcomes. As research continues, there is a need to conduct further studies to better understand and evaluate individuals' use of the internet and social media to look for health information. The results of the study can create an opportunity for policymakers to develop policies for more effective online health interventions that can help in changing health behaviors. Linking health systems to online platforms can ensure disseminating reliable online health information by trustworthy sources, which could contribute to improving health behaviors among adults in the United States in a cost-effective manner. 


\section{Additional Information \\ Disclosures}

Human subjects: All authors have confirmed that this study did not involve human participants or tissue. Animal subjects: All authors have confirmed that this study did not involve animal subjects or tissue. Conflicts of interest: In compliance with the ICMJE uniform disclosure form, all authors declare the following: Payment/services info: All authors have declared that no financial support was received from any organization for the submitted work. Financial relationships: All authors have declared that they have no financial relationships at present or within the previous three years with any organizations that might have an interest in the submitted work. Other relationships: All authors have declared that there are no other relationships or activities that could appear to have influenced the submitted work.

\section{References}

1. Maheri A, Tol A, Sadeghi R: Assessing the effect of an educational intervention program based on Health Belief Model on preventive behaviors of internet addiction. J Educ Health Promot. 2017, 6:63. 10.4103/jehp.jehp_129_15

2. Hamm MP, Chisholm A, Shulhan J, Milne A, Scott SD, Given LM, Hartling L: Social media use among patients and caregivers: a scoping review. BMJ Open. 2013, 3:e002819. 10.1136/bmjopen-2013-002819

3. Tennant B, Stellefson M, Dodd V, Chaney B, Chaney D, Paige S, Alber J: eHealth literacy and Web 2.0 health information seeking behaviors among baby boomers and older adults. J Med Internet Res. 2015, 17:e70. 10.2196/jmir.3992

4. Hoffman AS, Volk RJ, Saarimaki A, et al.: Delivering patient decision aids on the Internet: definitions, theories, current evidence, and emerging research areas. BMC Med Inform Decis Mak. 2013, 13:S13. 10.1186/1472-6947-13-S2-S13

5. Ahadzadeh AS, Pahlevan Sharif S, Ong FS, Khong KW: Integrating health belief model and technology acceptance model: an investigation of health-related internet use. J Med Internet Res. 2015, 17:e45. 10.2196/jmir.3564

6. Amante DJ, Hogan TP, Pagoto SL, English TM, Lapane KL: Access to care and use of the Internet to search for health information: results from the US National Health Interview Survey. J Med Internet Res. 2015, 17:e106. 10.2196/jmir.4126

7. Kontos E, Blake KD, Chou WY, Prestin A: Predictors of eHealth usage: insights on the digital divide from the Health Information National Trends Survey 2012. J Med Internet Res. 2014, 16:e172. 10.2196/jmir.3117

8. Xiao N, Sharman R, Rao HR, Upadhyaya S: Factors influencing online health information search: an empirical analysis of a national cancer-related survey. Decis Support Syst. 2014, 57:417-427. 10.1016/j.dss.2012.10.047

9. Caiata-Zufferey M, Abraham A, Sommerhalder K, Schulz PJ: Online health information seeking in the context of the medical consultation in Switzerland. Qual Health Res. 2010, 20:1050-1061. 10.1177/1049732310368404

10. Lee K, Hoti K, Hughes JD, Emmerton L: Dr Google and the consumer: a qualitative study exploring the navigational needs and online health information-seeking behaviors of consumers with chronic health conditions. J Med Internet Res. 2014, 16:e262. 10.2196/jmir.3706

11. Hackman CL, Pember SE: Qualitative investigation of health information seeking behavior utilizing social media in a college population. Am J Health Stud. 2016, 31:11-22.

12. Korda H, Itani Z: Harnessing social media for health promotion and behavior change . Health Promot Pract. 2013, 14:15-23. 10.1177/1524839911405850

13. Glanz K, Rimer BK, Viswanath K: Health Behavior: Theory, Research, and Practice. Glanz K, Rimer BK, Viswanath K (ed): Jossey-Bass, San Francisco, CA; 2015.

14. Health Information National Trends Survey 5 (HINTS 5) . (2017). Accessed: October 26, 2020: https://hints.cancer.gov/docs/methodologyreports/HINTS5_Cycle_2_Methodology_Report.pdf.

15. Robertson-Lang L, Major S, Hemming H: An exploration of search patterns and credibility issues among older adults seeking online health information. Can J Aging. 2011, 30:631-645. 10.1017/S071498081100050X

16. Chou WY, Prestin A, Lyons C, Wen KY: Web 2.0 for health promotion: reviewing the current evidence . Am J Public Health. 2013, 103:e9-18. 10.2105/AJPH.2012.301071

17. Renahy E, Parizot I, Chauvin P: Determinants of the frequency of online health information seeking: results of a web-based survey conducted in France in 2007. Inform Health Soc Care. 2010, 35:25-39. 10.3109/17538150903358784

18. Kim J, Park HA: Development of a health information technology acceptance model using consumers' health behavior intention. J Med Internet Res. 2012, 14:e133. 10.2196/jmir.2143

19. Campbell AJ, Ridout BF, Linden M, Collyer B, Dalgleish J: A preliminary understanding of search words used by children, teenagers and young adults in seeking information about depression and anxiety online. J Technol Hum Serv. 2018, 36:1-14. 10.1080/15228835.2018.1518186

20. Lagoe C, Atkin D: Health anxiety in the digital age: an exploration of psychological determinants of online health information seeking. Comput Hum Behav. 2015, 52:484-491. 10.1016/j.chb.2015.06.003

21. Graffigna G, Barello S, Bonanomi A, Riva G: Factors affecting patients' online health information-seeking behaviours: the role of the Patient Health Engagement (PHE) Model. Patient Educ Couns. 2017, 100:19181927. 10.1016/j.pec.2017.05.033

22. Tate DF, Lyons EJ, Valle CG: High-tech tools for exercise motivation: use and role of technologies such as the internet, mobile applications, social media, and video games. Diabetes Spectr. 2015, 28:45-54. 10.2337/diaspect.28.1.45

23. Shahab L, Brown J, Gardner B, Smith SG: Seeking health information and support online: does it differ as a function of engagement in risky health behaviors? Evidence from the health information national trends survey. J Med Internet Res. 2014, 16:e253. 10.2196/jmir.3368 